\title{
Kinematics of Giant Low Surface Brightness Galaxies
}

\author{
T. E. Pickering
}

Kapteyn Institute, Postbus 800, 9700 AV Groningen, The Netherlands

\begin{abstract}
High sensitivity $\mathrm{H}$ I observations now exist for six giant low surface brightness (LSB) disk galaxies including the two prototypes, Malin 1 (Bothun et al. 1987; Impey \& Bothun 1989) and F568-6 (also known as Malin 2; Bothun et al. 1990). Their H I surface brightnesses are generally low, but proportionally not as low as their optical surface brightnesses. Their total $\mathrm{H}$ I masses and radial extents are quite large, however, with $M_{\mathrm{HI}} \sim 10^{10} M_{\odot} h_{75}^{-2}$ and with detectable $\mathrm{H}$ I out to $100 \mathrm{kpc}$ $h_{75}^{-1}$ or more in a couple of cases. The rotation curves of these systems rise slowly and are consistent with negligible disk contribution, similar to many previously observed dwarf galaxies. However, the peak rotation velocities of these galaxies are high $\left(>200 \mathrm{~km} \mathrm{~s}^{-1}\right)$ and infer high dynamical masses. These galaxies provide the some of the first examples of galaxies that are both massive and dark matter dominated.
\end{abstract}

\section{Introduction}

The serendipitous discovery of Malin 1, whose remarkable properties are described by Bothun et al. (1987) and Impey \& Bothun (1989), demonstrated the existence of very large, very luminous, but very diffuse, galaxies. As an example of an $L^{*}$ galaxy that is extremely hard to detect, Malin 1 helped confirm the idea, first clearly articulated by Disney (1976), that the brightness of the night sky can and does bias our understanding of galaxies and of the range of galaxy properties. It also helped to spur subsequent deliberate searches for low surface brightness (LSB) galaxies such as those of Schombert \& Bothun (1988) and Impey et al. (1996). While these surveys didn't find any objects with properties as extreme as Malin 1 (indeed, none of these surveys was deep enough to detect Malin 1 itself), they did turn up several giant LSB galaxies whose properties approach those of Malin 1. F568-6 (Bothun et al. 1990), 1226+0105 (Sprayberry et al. 1993), UGC 6614 (van der Hulst et al. 1993), and two other galaxies from Sprayberry et al. (1995a) are the closest "cousins" to Malin 1 and have physically large disks with scale lengths greater than $10 \mathrm{kpc} h_{75}^{-1}$, total luminosities of $L^{*}$ or greater, central surface brightnesses in $B$ fainter than $23 \mathrm{mag} \mathrm{arcsec}^{-2}$, and total $\mathrm{H}$ I masses of greater than $10^{10} M_{\odot} h_{75}^{-2}$.

These giant LSB galaxies probe a previously unexplored region of parameter space for galactic disks. Knezek (1993) and Sprayberry et al. (1995b) have tabulated optical and single-dish HI properties, while previous H I synthesis studies of LSBs by McGaugh (1992), van der Hulst et al. (1993), de Blok et al. (1996), and de Blok \& McGaugh (1997) concentrated largely on smaller, less 
luminous LSB galaxies. Rotation curve studies so far have shown that the shape of a rotation curve is closely related to the rotation velocity of the galaxy. Slow rotators (i.e., the dwarfs mentioned above) have slowly rising rotation curves. Galaxies with rotation speeds in excess of $100 \mathrm{~km} \mathrm{~s}^{-1}$ tend to have flat rotation curves while the fastest rotators $\left(>200 \mathrm{~km} \mathrm{~s}^{-1}\right)$ have slightly declining rotation curves (Casertano \& van Gorkom 1991; Broeils 1992). These results for the faster rotators are entirely based on HSB galaxies, however.

In the inner parts of HSB galaxies the rotation curves are often dominated by the luminous mass, i.e., the rotation curve can be fit by scaling the luminous component by a constant mass-to-light ratio (the "maximal disk" hypothesis). Only at large radii does the dark matter component become important. However, within the massive HSB galaxies a slight trend was found with surface brightness: less bright, less compact galaxies tend to be more dark matter dominated (Casertano \& van Gorkom 1991). At a given total mass and mass-to-light ratio, a disk with a larger scale length will reach it's peak rotation velocity at a larger radius and will have a lower peak rotation velocity than the disk with the smaller scale length. If the galaxies have comparable halo properties, the halo should contribute proportionally more to the kinematics in the large scale length case. With their much larger and thus more diffuse disks giant LSB galaxies provide an important contrast to massive HSB galaxies and an important probe into the structure of galactic halos.

\section{The Data}

To date we have obtained VLA H I synthesis observations for five giant LSB galaxies. The data for the first four including Malin 1 and F568-6 have been published in Pickering et al. (1997) and the fifth, UGC 2936, has been published as part of my thesis work, Pickering (1998). The $\mathrm{H}$ I velocity fields overlayed on $\mathrm{HI}$ intensity maps for these five galaxies are shown in Figure 1. Australia Telescope Compact Array H I data for another giant, gas-rich LSB galaxy, NGC 289, has been published by Walsh et al. (1997).

Figure 2 shows the radially-averaged $\mathrm{H}$ I surface density profiles, derived rotation curves, and critical density profiles calculated from the rotation curves for the galaxies from Pickering et al. (1997) and Pickering (1998). The critical density profiles are calculated using the method of Kennicutt (1989) assuming a gas velocity dispersion of $6 \mathrm{~km} \mathrm{~s}^{-1}$.

\section{Stability of Giant LSB Disks}

In two of the cases shown in Figure 2 the $\mathrm{H}$ I surface density lies below the critical density at all radii which is consistent with the low rate of star formation in those disks. However, in the other three cases significant portions of the disks have $\mathrm{H} \mathrm{I}$ surface densities that exceed the critical density. In the case of UGC 2936 there is a fairly significant amount of star formation occurring. Figure 3 shows the optical rotation curve based on $\mathrm{H} \alpha$ for UGC 2936 plotted with the $\mathrm{H}$ I positionvelocity map and rotation curve. The last measured points in the optical rotation curve occur at radii of about $14 \mathrm{kpc} h_{75}^{-1}$ which is in excellent agreement with the radii at which the $\mathrm{H}$ I surface density shown in Figure 2 falls below the critical 

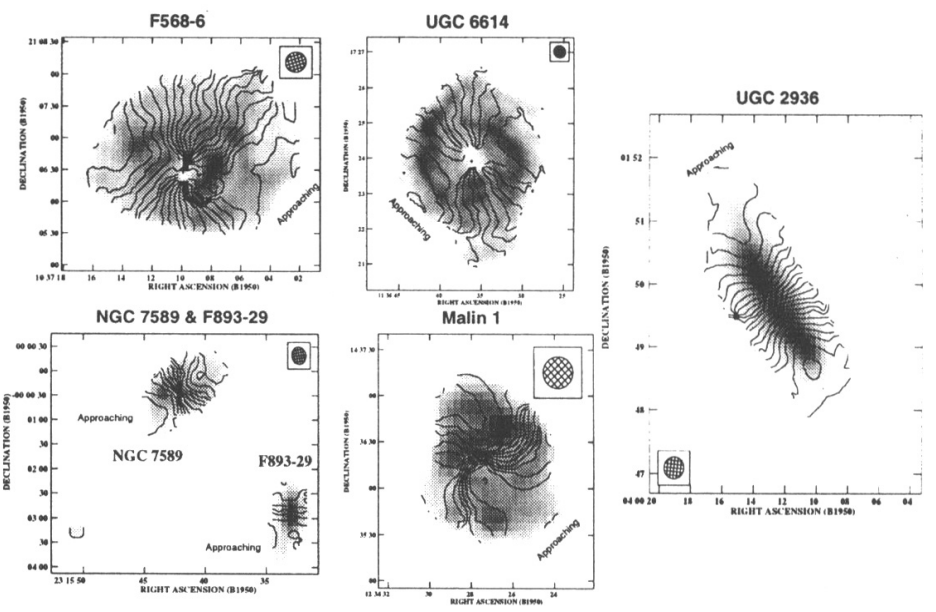

Figure 1. $\mathrm{H}$ I isovelocity contours overlayed on $\mathrm{H}$ I intensity greyscale for 5 giant LSB galaxies: Malin 1, F568-6, UGC 6614, NGC 7589, and UGC 2936 . The velocity contour spacing is $20 \mathrm{~km} \mathrm{~s}^{-1}$ in all cases.
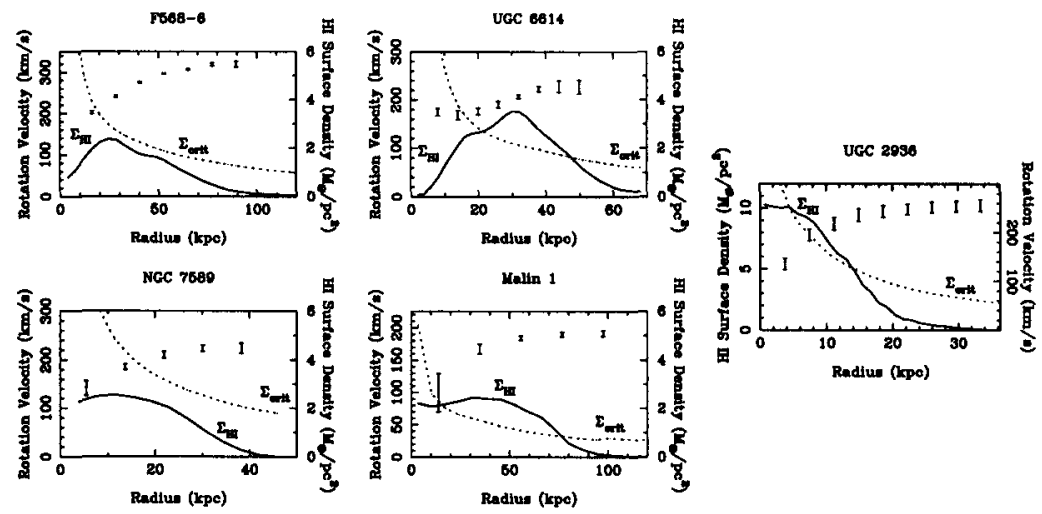

Figure 2. Radially-averaged H I surface density profiles (solid lines), rotation curves (data points), and critical density profiles (dotted lines) for Malin 1, F568-6, UGC 6614, NGC 7589, and UGC 2936. The critica! density profiles are calculated assuming a gas velocity dispersion of 6 $\mathrm{km} \mathrm{s}^{-1}$. 


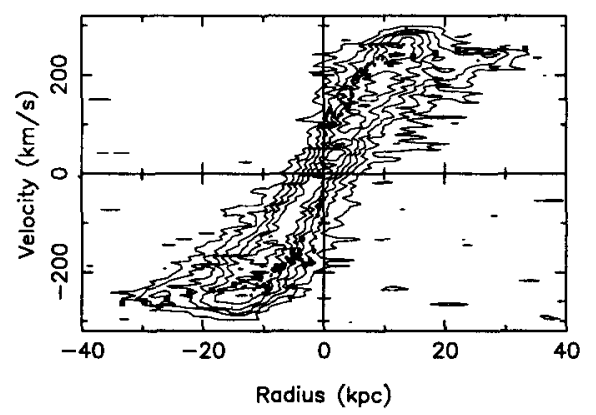

Figure 3. Optical rotation curve (filled circles) for UGC 2936 plotted with the H I rotation curve (filled squares) and major axis positionvelocity map (contours). The position-velocity map is corrected for

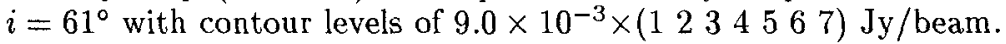

density. In the case of Malin 1 the large uncertainty in the inclination, and thus the absolute scaling of the rotation curve, leads to a large uncertainty in the determination of the critical density as well as in the line-of-sight corrections to the surface density. The inclination is much more well-determined in the case of UGC 6614 , though, so it's more clearly a case where the critical density criterion predicts widespread star formation where none is observed to occur. This is an indication that the conditions in a giant LSB disk are quite different than in the HSB galaxies used to calibrate the critical density criterion. One might expect this to be the case since the dark halo will be more kinematically dominant as a disk becomes more diffuse resulting in a lower restoring force in the disk. The lower restoring force results in a thicker and more stable disk at a given velocity dispersion which might explain the results for UGC 6614.

\section{Mass-to-Light Ratios in Giant LSB Galaxies}

If these rotation curves are modeled by a disk component alone, the amplitude of the rotation curves in these galaxies requires $R$-band $M / L$ 's (assuming $h_{75}=1$ ) of up to 15-20 in the cases of F568-6 and UGC 6614. The shapes of the curves, however, are not well-produced by the disk-only fits. Quillen \& Pickering (1997) used the strengths of the optical and $\mathrm{H} \mathrm{I}$ spiral arms along with the strengths of the velocity perturbations due to the spiral arms to place much better constraints on the disk $M / L$ 's in UGC 6614 and F568-6. They find that in both cases the $R$ band $M / L$ 's are in the range of about 1 to 5 which clearly rule out the maximal disk-only results. If the bulge component is included and assumed to have the same $M / L$ as the disk, the $M / L$ constraints are even tighter: < 1.5 for F568-6 and $<2.5$ for UGC 6614 . Thus there's no indication that giant LSB galaxies have disk mass-to-light ratios that differ significantly from more normal HSB galaxies.

The global $M / L$ 's for giant LSB galaxies derived by measuring the mass enclosed by the last measured points in the rotation curves are similarly not far out of line with what is found for HSB galaxies. This is rather remarkable since their last measured points are at radii approaching $100 \mathrm{kpc} h_{75}^{-1}$ in a couple of 


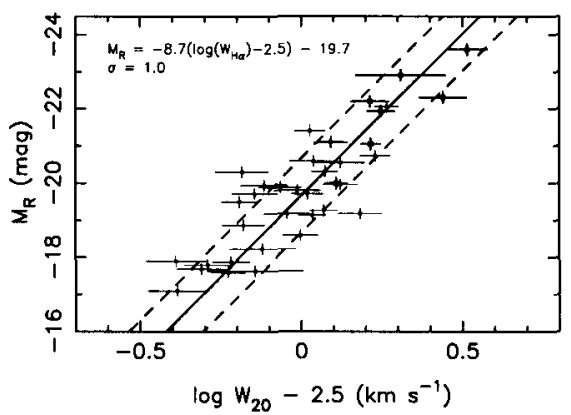

Figure 4. Absolute $R$ magnitude, $M_{R}$, versus $\mathrm{H} \mathrm{I}$ linewidth, $W_{20}$ for the giant LSB galaxies (filled circles) and other LSB galaxies from the samples of Zwaan et al. (1995) and Sprayberry et al. (1995b) (filled squares).

cases and greater than $35 \mathrm{kpc} h_{75}^{-1}$ in all cases. The five galaxies from Pickering et al. (1997) and Pickering (1998) all have $R$-band global $M / L$ 's in the range 11-15. A typical HSB galaxy such as NGC 3198 (Begeman 1989), for example, has a total $R$-band $M / L$ of 10 at a radius of $22 \mathrm{kpc} h_{75}^{-1}$. Zwaan et al. (1995) and Sprayberry et al. (1995b) showed that LSB galaxies follow the same TullyFisher relation as comparable HSB galaxies which also implies that their global mass-to-light ratios must be at least roughly similar. Figure 4 shows the results for the giant LSBs plotted with results for other LSB galaxies. The giant LSB galaxies are completely consistent within the uncertainties with the Tully-Fisher relation derived from the other LSB galaxies.

\section{Shapes of Giant LSB Rotation Curves and NFW Halos}

The high resolution numerical simulations of galactic halos by Navarro et al. (1996) showed that dark matter halos over several orders of magnitude in mass have density profiles that can all be well-described by a single two parameter density profile. This so-called NFW halo profile has a halo circular velocity profile of the form

$$
V_{\mathrm{NFW}}(r)=V_{200} \sqrt{\frac{1}{x} \frac{\ln (1+c x)-c x /(1+c x)}{\ln (1+c)-c /(1+c)}}
$$

where the two free parameters are $V_{200}$, the circular velocity of the halo at the virial radius, and $c$, the concentration of the halo. Navarro et al. (1996) compared some observed rotation curve shapes with those predicted by a NFW halo profiles within a Standard Cold Dark Matter (SCDM) universe and found that while HSB galaxies can be made consistent by tweaking $M / L$, LSB dwarfs cannot be made consistent with SCDM.

Navarro et al. (1997) developed this further and showed that the NFW halo profile is a generic result of hierarchical clustering and that different cosmologies result in different relations between $V_{200}$ and $c$. Using this, one can turn the comparison around and see what cosmologies are consistent with the values of 


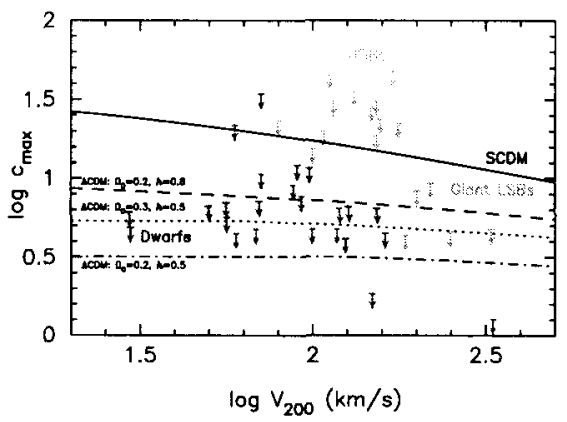

Figure 5. Results of maximal NFW fits to samples of LSB and HSB rotation curves taken from the literature plotted with the predicted $V_{200}-c$ relations for different cosmologies. The predictions were calculated using the method described by Navarro et al. (1997)

$V_{200}$ and $c$ one measures from galactic rotation curves. A quick and dirty way to estimate this and place upper limits on $c$ is to fit galactic rotation curves using only Equation 1, basically a maximal NFW halo fit. The results of some maximal halo fits to the giant LSB galaxies as well as other samples of LSB and HSB rotation curves taken from the literature are shown in Figure 5. As in Navarro et al. (1996), the HSB galaxies could be made consistent with SCDM. They could also be made consistent with everything else and hence don't have much predictive power when fitting with a halo only. This is because much of the mass concentration in HSB galaxies is due to the baryonic component rather than the dark halo component. In the LSB cases the baryonic component is much less concentrated and less kinematically important so they offer better constraints on the halo properties. Almost all of those cases fall below and are not consistent with SCDM. Most of them are consistent with lower density models that include a cosmological constant $(\Lambda \mathrm{CDM})$. However, a few have very low best-fit concentrations when fitted with an NFW halo only. Those are cases where the rotation curve is solid-body-like and linearly rising and thus has a shape that is not well-described by Equation 1.

\section{Conclusions}

Giant LSB galaxies are some of the first known examples of massive galaxies whose kinematics are dark halo dominated at almost all radii. Their rotation curve shapes cannot be reproduced by scaling their stellar components by constant mass-to-light ratios. Even the amplitudes of their rotation curves would require higher mass-to-light ratios than are allowed by other constraints such as spiral arm strengths. The shapes of their rotations curves cannot be made consistent with the predictions of SCDM, but are consistent with the predictions of low density $\Lambda$ CDM models.

While at a given radius a giant LSB galaxy's halo dominates its kinematics to a greater extent than in an HSB, the global mass-to-light ratios are about the same in both cases. The giant LSB galaxies also follow, or are at least consistent with, the same Tully-Fisher relation as other LSB and HSB galaxies. 


\section{References}

Begeman, K. G. 1989, A\&A, 223, 47

Broeils, A. H. 1992, Ph.D. thesis, University of Groningen

de Blok, W. J. G., McGaugh, S. S., \& van der Hulst, J. M. 1996, MNRAS, 283, 18

de Blok, W. J. G., \& McGaugh, S. S. 1997, MNRAS, 290, 533

Bothun, G. D., Impey, C. D., Malin, D. F., \& Mould, J. R. 1987, AJ, 94, 23

Bothun, G. D., Schombert, J. M., Impey, C. D., \& Schneider, S. E. 1990, ApJ, 360,427

Casertano, S., \& van Gorkom, J. H. 1991, AJ, 101, 1231

Disney, M. J. 1976, Nature, 263, 573

Impey, C. D., \& Bothun, G. D. 1989, ApJ, 341, 89

Impey, C. D., Sprayberry, D., Irwin, M. J., \& Bothun, G. D. 1996, ApJS, 105 , 209

Kennicutt, R. C. 1989, ApJ, 344, 685

Knezek, P. M. 1993, Ph.D. thesis, University of Massachusetts

McGaugh, S. S. 1992, Ph.D. thesis, University of Michigan

Navarro, J. F., Frenk, C. S., \& White, S. D. M. 1996, ApJ, 462, 563

Navarro, J. F., Frenk, C. S., \& White, S. D. M. 1997, ApJ, 490, 493

Pickering, T. E., Impey, C. D., van Gorkom, J. H., \& Bothun, G. D. 1996, AJ, 114,1858

Pickering, T. E. 1998, Ph.D. thesis, University of Arizona

Quillen, A., \& Pickering, T. E. 1997, AJ, 113, 2075

Schombert, J. S., \& Bothun, G. D. 1988, AJ, 95, 1389

Sprayberry, D., Impey, C. D., Irwin, M. J., McMahon, R. G., \& Bothun, G. D. 1993, ApJ, 417, 114

Sprayberry. D., Impey, C. D., Bothun, G. D., \& Irwin, M. J. 1995a, AJ, 109, 558

Sprayberry, D., Bernstein, G. M., Impey, C. D., \& Bothun, G. D. 1995b, ApJ, 438,72

van der Hulst, J. M., Skillman, E. D., Smith, T. R., Bothun, G. D., McGaugh, S. S., \& de Blok, W. J. G. 1993, AJ, 106, 548

Walsh, W., Staveley-Smith, L., \& Oosterloo, T. 1997, AJ, 113, 1591

Zwaan, M. A., van der Hulst, J. M., de Blok, W. J. G., \& McGaugh, S. S. 1995, MNRAS, 273, L35 